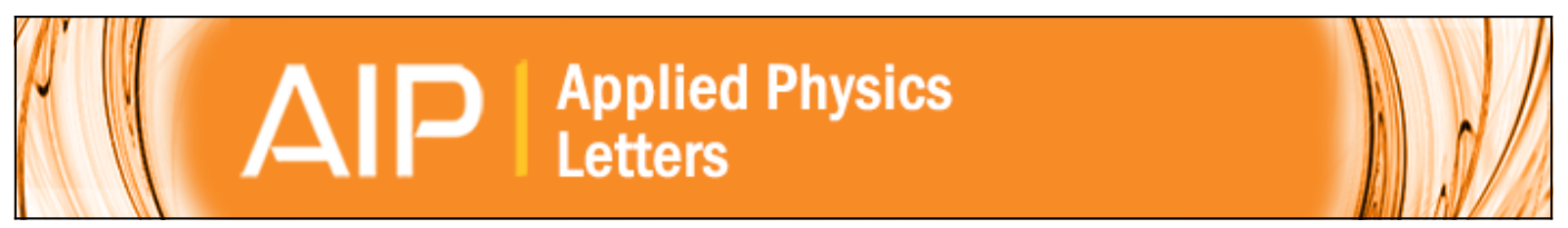

\title{
Grain boundaries in granular materials-A fundamental limit for thermal stability
}

L. Saharan, C. Morrison, Y. Ikeda, K. Takano, J. J. Miles, T. Thomson, T. Schrefl, and G. Hrkac

Citation: Applied Physics Letters 102, 142402 (2013); doi: 10.1063/1.4801316

View online: http://dx.doi.org/10.1063/1.4801316

View Table of Contents: http://scitation.aip.org/content/aip/journal/apl/102/14?ver=pdfcov

Published by the AIP Publishing

\section{Articles you may be interested in}

Frequency dependence of critical switching asteroid of $\mathrm{CoCrPt-SiO} 2$ granular film under 50-ns microwave impulse

J. Appl. Phys. 114, 043915 (2013); 10.1063/1.4816778

Influence of intergranular exchange coupling on the magnetization dynamics of CoCrPt:SiO2 granular media J. Appl. Phys. 112, 033918 (2012); 10.1063/1.4745906

Recording performance and thermal stability in perpendicular media with enhancement of grain isolation as well as magnetic anisotropy field

J. Appl. Phys. 111, $07 B 705$ (2012); 10.1063/1.3677307

Magnetic anisotropy derived from interparticle interactions in $\mathrm{CoPtCr} / \mathrm{SiO} 2$ granular thin film longitudinal media J. Appl. Phys. 93, 2804 (2003); 10.1063/1.1542653

Improved thermal stability of synthetic ferrimagnetic media with enhanced exchange coupling strength Appl. Phys. Lett. 80, 2719 (2002); 10.1063/1.1467696

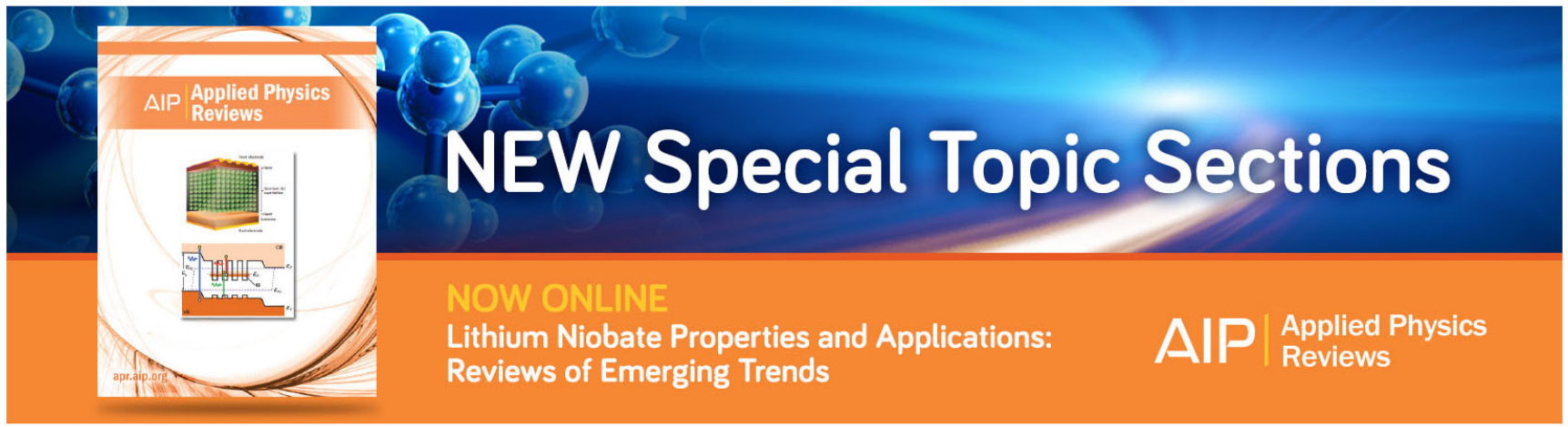




\title{
Grain boundaries in granular materials-A fundamental limit for thermal stability
}

\author{
L. Saharan, ${ }^{1}$ C. Morrison, ${ }^{2}$ Y. Ikeda, ${ }^{3}$ K. Takano, ${ }^{3}$ J. J. Miles, ${ }^{2}$ T. Thomson, ${ }^{2}$ T. Schrefl, ${ }^{4}$ \\ and G. Hrkac ${ }^{1,5}$ \\ ${ }^{1}$ Department of Engineering Materials, University of Sheffield, Sheffield S1 3JD, United Kingdom \\ ${ }^{2}$ School of Computer Science, University of Manchester, Manchester M13 9PL, United Kingdom \\ ${ }^{3}$ San Jose Research Center, HGST, San Jose, California 95135, USA \\ ${ }^{4}$ St. Pölten University of Applied Science, Matthias Corvinus Straße 15, St. Pölten A-3100, Austria \\ ${ }^{5}$ College of Engineering, Mathematics and Physical Sciences, University of Exeter, Devon, United Kingdom
}

(Received 8 February 2013; accepted 26 March 2013; published online 8 April 2013)

\begin{abstract}
We show that thermal-stability and the associated switching field in well segregated, nanoscale granular materials is explained by grain boundary and interface effects. Grain boundaries pose a fundamental limit on scaling rooted in their chemical and morphological structure, while exchange interactions across interfaces cause the switching to deviate from the expected coherent Stoner-Wohlfarth behaviour. Measurements and simulations of CoCrPt-systems show a clear shift in applied field angle behaviour, arising from exchange-coupling between magnetic-phases, while the quantitative switching field can only be explained by a transition layer surrounding the grains. These results are potentially significant for Heat-Assisted-Magnetic Recording and Bit-Patterned-Media Recording. @ 2013 AIP Publishing LLC. [http://dx.doi.org/10.1063/1.4801316]
\end{abstract}

A prerequisite to increase the performance of granular magnetic materials such as reduced rare-earth sintered/ nano-magnets and recording media is a fundamental understanding of their magnetization reversal behaviour. The key physical phenomena are spin-orbit coupling effects (exchange coupling and magneto-crystalline anisotropy), structural complexity (lattice defects/strains), and thermal activation. Thermal effects and inter/intra exchange coupling have been studied experimentally and theoretically ${ }^{1-6}$ but the behaviour of many systems cannot be explained by these effects alone. It was previously shown that the switching behavior of twophase perpendicular granular exchange coupled systems ${ }^{4,7}$ can deviate from a coherent, Stoner-Wohlfarth type behaviour, ${ }^{8}$ towards a Kondorsky-like model, ${ }^{9}$ which is revealed through the angular dependence of remanent coercivity. One possible source of incoherency arises from an increased degree of lateral inter-granular exchange coupling, rather than from the intra-granular exchange spring. ${ }^{5}$ An alternative explanation was deduced from experiments at elevated temperature, showing that a decrease in the depth of the StonerWohlfarth minimum can arise from thermal activation. ${ }^{6}$ However, it has been demonstrated that a shift in the angle of applied field at which the minimum switching field occurs is caused by incoherent reversal induced by inter-granular exchange coupling or incoherency within larger grains, rather than thermal activation. ${ }^{10,11}$ In order to understand how these different phenomena contribute to the experimental observations, it is essential to understand the roles of the coupling mechanisms intrinsic to individual grains, such as the interface between two magnetic phases and the region of reduced magnetisation at the grain boundary.

To study these interface effects, we choose a well segregated granular CoCrPt-oxide two-phase magnetic system that provides an ideal platform to study reversal in granular magnets both experimentally and through simulation. We report a systematic study of the switching field and angle dependence of reversal in CoCrPt-oxide films as a function of interlayer exchange and grain boundaries at $292 \mathrm{~K}$. We compare the predictions of a finite temperature micromagnetic model of a single, isolated two-phase $\mathrm{CoCrPt}$ grain to measurements, which allows us to explore the effect of grain boundaries (boundary region of a grain at the surface) and interlayer exchange coupling on the qualitative and quantitative switching behaviour. It is shown that the introduction of an exchange transition layer between two magnetic phases and the presence of grain boundaries are essential to explain the switching field behaviour as a function of angle and the experimentally observed values of the switching fields.

The granular recording medium studied here was deposited by dc magnetron sputtering onto a $65 \mathrm{~mm}$ glass disk substrate. A $30 \mathrm{~nm}$ CoFe based soft underlayer (SUL) was deposited, followed by a Ru seed layer. Next, a CoCrPt-SiO granular, segregated recording layer of $11 \mathrm{~nm}$ was deposited, followed by a CoCrPt-based alloy layer of $6 \mathrm{~nm}$. Finally, the sample was finished with a protective overcoat and a layer of lubricant.

A Microsense Model 10 vector vibrating sample magnetometer (VSM) was used to measure remanence curves as a function of angle from the film normal. An in-plane pinning field was applied while measuring the remanent magnetization out-of-plane, to allow the contribution of the SUL to be subtracted from the measurement. ${ }^{8}$ The remanent coercivity $H_{c r}$ was determined from each remanence curve, allowing a plot of $H_{c r}$ to be produced as a function of angle.

As outlined above, the magnetic medium is a complex multi-layered structure $^{12}$ where the ferromagnetic core regions are embedded inside a non-magnetic $\mathrm{SiO}_{2}$ matrix. Due to the intermixing of the ferromagnetic material with the $\mathrm{SiO}_{2}$, the size of the ferromagnetic region is found to be smaller than the corresponding grain size. It was shown by experiments ${ }^{12}$ that in the boundary region of the ferromagnetic grain (grain boundary), the magnetic properties show a 
change at the interface between the $\mathrm{CoCrPt}$ and the $\mathrm{SiO}_{2}$. In the simplest case, we consider this change as a step at the interface that divides the grains into different regions based on magnetic properties: the magnetic core and the grain boundary region. The magnetic core is concentrated in the centre of the grain surrounded by the less magnetic boundary region. ${ }^{12}$

One of the main aims of this study is to explore the magnetic structure and magnetization reversal of exchange coupled composite media to determine the effects of intergranular interfaces and grain boundaries on the intrinsic coercivity of high anisotropy materials. In order to understand these influences, finite temperature micromagnetic simulations were undertaken to study the reversal process and the switching field as a function of an exchange break interlayer with/without grain boundaries. The study was divided into three model systems, uniquely designed to explore either the switching field effects or the angular switching field dependency. The calculations were performed at a finite temperature of $292 \mathrm{~K}$ with an attempt frequency $f_{o}$ of $10^{11}$ $\mathrm{Hz}$ and a simulated measurement time $t$ of $10 \mathrm{~s}$, using the nudged elastic band method (NEB) ${ }^{13}$ combined with a micromagnetic model where we simultaneously solve the static Maxwell equations and the Landau-Lifshitz-Gilbert equation with a hybrid finite element/boundary element method. ${ }^{14}$

In the finite element simulations, we use a graded mesh scheme with sub-nanometer resolution near the interfaces. This approach provides a good description of our system as Kronmüller ${ }^{15}$ showed that for hard magnets with narrow domain wall widths, both atomistic models and a continuum models give similar results, although domain walls tend to be narrower in the atomistic model. Local variations in the intrinsic material properties can be accounted on an element by element basis. Based on the experimental measurements done by Lister et al., ${ }^{12}$ we assumed a stepwise-reduced anisotropy over a certain volume. The experiments strongly suggest that the composition is different at the grain boundary and disordered, meaning that one has different lattice structures based on composition with a reduced crystalline anisotropy.

For the nudge elastic band method, mainly the volume and its associated material properties contribute to the energy barrier from which we calculate the switching field. Suess et al. ${ }^{16}$ showed that the switching field of grains mainly depends on the grain volume or grain diameter and show that it is possible to describe a magnetic granular film by a representative grain with an effective grain diameter derived from the grain size distribution of the film. Like in the work of Suess et al. ${ }^{16}$ we use an effective grain diameter in our simulations that is derived from a grain size distribution from experimental measurements.

To determine switching probability, we first initialise the micromagnetic model with the magnetization parallel to the easy axis (normalized to $m_{z}=+1$ ) and run the model at $\mathrm{T}=0 \mathrm{~K}$ to determine the nearest stable state, which is taken to be the initial state prior to thermally activated reversal. We then repeat the process to determine the reversed state by initialising the grain magnetization in the reverse direction (normalized to $m_{z}=-1$ ). In the next step, the energy barrier between the two previously calculated stable states is computed using the NEB method. This energy barrier is determined using this method for each applied field magnitude at the given field angle. The process is then repeated for each field angle calculated.

In the model, cylindrical grains have the structure (along the z-direction) of hard phase/exchange transition phase/soft phase with $11 / 0.5 / 6 \mathrm{~nm}$ thicknesses and a diameter of $8 \mathrm{~nm}$. The three structures simulated here are derived from polarized small angle neutron scattering (SANSpol) experiments by Lister $e t$ al. ${ }^{12}$ with the magnetic parameters derived from vibrating sample magnetometry measurements at a temperature of $292 \mathrm{~K}$. A graphical schematic of each of the model systems is shown as an inset in Figures 1-3.

In the first model, we chose a homogenous magnetic grain structure. This provides simulation results, which show how a perfect interface and grain structure would behave as a reference structure for comparison with more complex structures.

The grain has material properties as follows: hard phase: crystalline anisotropy $K_{\text {hard }}=5.86 \times 10^{5} \mathrm{~J} / \mathrm{m}^{3}$, magnetic polarization $J_{\mathrm{s}}=0.90 \mathrm{~T}$, and exchange constant of $A=1 \times 10^{-11} \mathrm{~J} / \mathrm{m}$. Soft phase: $K_{\text {soft }}=1.74 \times 10^{5} \mathrm{~J} / \mathrm{m}^{3}, J_{\mathrm{s}}=0.57 \mathrm{~T}$, and $A=$ $1 \times 10^{-11} \mathrm{~J} / \mathrm{m}$. Exchange transition phase: $K=1.74 \times 10^{5} \mathrm{~J} / \mathrm{m}^{3}$, $J_{\mathrm{s}}=0.57 \mathrm{~T}$, and $A=0.4 \times 10^{-11} \mathrm{~J} / \mathrm{m}$.
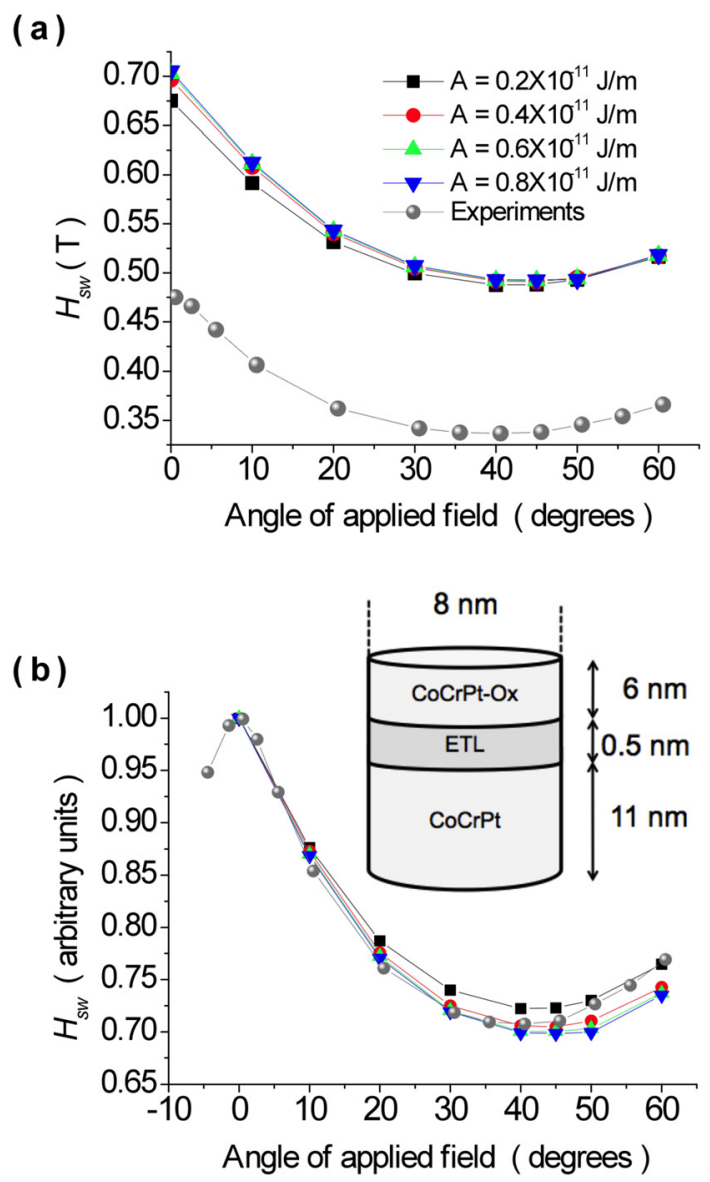

FIG. 1. Comparison of simulated (a) switching field and (b) normalized switching field as a function of angle for the CoCrPt magnetic grain at $292 \mathrm{~K}$ with experiments. The value of the attempt frequency is $10^{11} \mathrm{~Hz}$ and the measurement time is $10 \mathrm{~s}$. The different markers of the curve represent the different values of the exchange in the exchange transition layer in the range of $0.2 \times 10^{-11} \mathrm{~J} / \mathrm{m}-0.8 \times 10^{-11} \mathrm{~J} / \mathrm{m}$. The grey dot curve is the corresponding experimental data. Inset shows the schematic of the simulated grain model. 


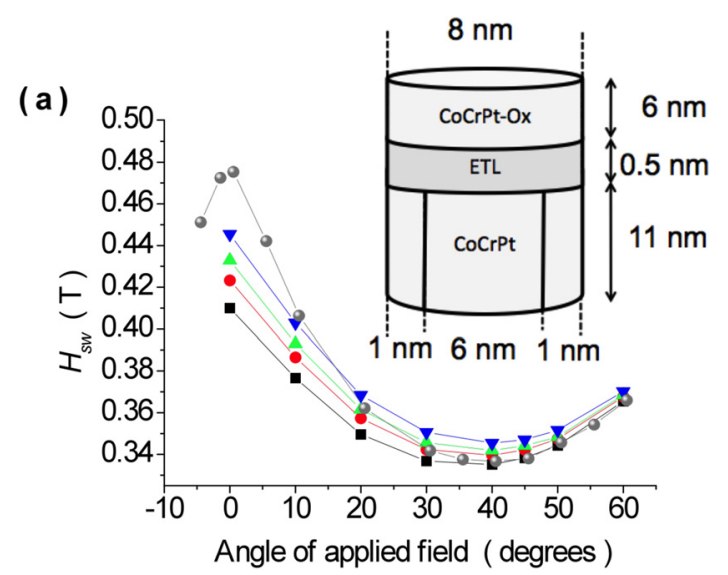

(b)

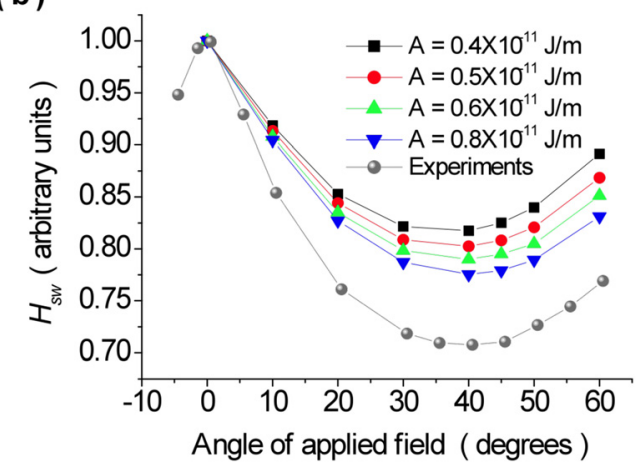

FIG. 2. Comparison of simulated (a) switching field and (b) normalized switching field as a function of angle for the CoCrPt magnetic grain at $292 \mathrm{~K}$ with experiments. The attempt frequency and measurement time used in calculations are $10^{11} \mathrm{~Hz}$ and $10 \mathrm{~s}$. The square curve represents the simulation values for an exchange value of the exchange transition layer of $0.4 \times 10^{-11} \mathrm{~J} / \mathrm{m}$, the dot curve for $0.5 \times 10^{-11} \mathrm{~J} / \mathrm{m}$, the up triangle curve for $0.6 \times 10^{-11} \mathrm{~J} / \mathrm{m}$, and the down triangle curve for $0.8 \times 10^{-11} \mathrm{~J} / \mathrm{m}$. The grey dot curve is the corresponding experimental data. Inset shows the schematic of the simulated grain model.

In Figure 1, we show a comparison with experiments of the switching field and normalised switching field for the exchange coupled composite grain. The comparison of the switching field in Figure 1(a) with different exchange transition layer exchange constants shows that the calculated switching field values are higher than the experimental values by $0.2 \mathrm{~T}$, at an applied field angle of $0^{\circ}$. However, apart from the discrepancy in the switching field magnitude, the trend in the switching field as a function of angle predicted by the model is similar to that found experimentally, as can be seen from the normalised values plotted in Figure 1(b).

The minimum switching field angle for the experimental curves is $40^{\circ}$, deviating from the Stoner-Wohlfarth model where the minimum angle lies at $45^{\circ}$. In the simulations, the minimum switching field angle is $45^{\circ}$ for high exchange values in the exchange transition layer ranging from $0.4 \times 10^{-11} \mathrm{~J} / \mathrm{m}$ to $0.8 \times 10^{-11} \mathrm{~J} / \mathrm{m}$, whereas for a low exchange of 0.2 $\times 10^{-11} \mathrm{~J} / \mathrm{m}$, the minimum switching field angle is $40^{\circ}$ with an absolute minimum switching field of $0.487 \mathrm{~T}$.

In the second model, we vary the magnetic structure of the exchange coupled composite grain (see inset Figure 2(a)). Based on the work of Lister et al., ${ }^{12}$ we approximate the gradual change of the magnetic composition of the hard phase across the grain boundary by dividing the hard phase into two parts and allocate different material properties to each of these

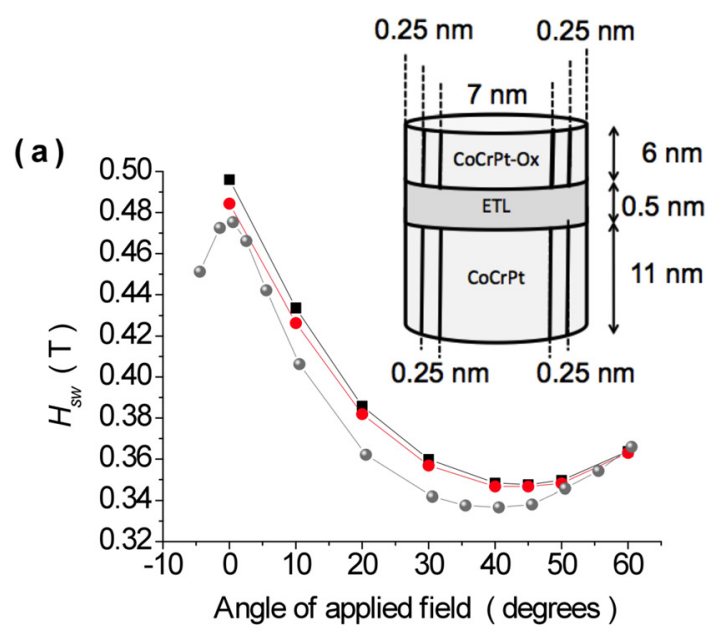

(b)

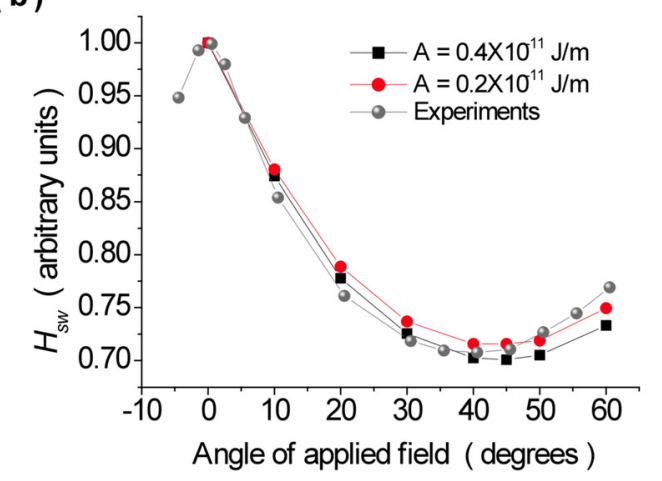

FIG. 3. Comparison of simulated (a) switching field and (b) normalized switching field as a function of angle for the CoCrPt exchange magnetic grain at $292 \mathrm{~K}$ with experiments. The attempt frequency and measurement time used in the calculations are $10^{11} \mathrm{~Hz}$ and $10 \mathrm{~s}$. The square curve represents the simulation values for an exchange value of the exchange transition layer of $0.4 \times 10^{-11} \mathrm{~J} / \mathrm{m}$ and the dot curve the ones for $0.2 \times 10^{-11} \mathrm{~J} / \mathrm{m}$. The grey dot curve is the corresponding experimental data. Inset shows the schematic of the simulated grain model.

sub volumes. The core has radius $3 \mathrm{~nm}$ with material properties identical to model 1 , the boundary has thickness $1 \mathrm{~nm}$ with an anisotropy that is $20 \%$ of $K_{\text {hard }}$. The exchange constant $A=1 \times 10^{-11} \mathrm{~J} / \mathrm{m}$ and magnetic polarization $J_{\mathrm{s}}=0.90 \mathrm{~T}$ throughout the hard phase. It is expected that the variation in the magnetic properties over the volume of the grain will alter the switching characteristics of the composite medium. Figure 2 compares the results of the simulation with experiments. In Figure 2(a), we show the switching field as a function of applied field angle for different exchange constants in the exchange transition layer ranging from $0.4 \times 10^{-11} \mathrm{~J} / \mathrm{m}-$ $0.8 \times 10^{-11} \mathrm{~J} / \mathrm{m}$ together with the experimental data. We see that the model predicts the switching field well at intermediate angles (30 to 60 degrees) but deviates slightly for small angles $\left(0^{\circ}\right.$ to $\left.30^{\circ}\right)$. Furthermore, the model also correctly predicts the minimum switching field angle, which is $40^{\circ}$ in agreement with experiments, see Figure 2(b).

Despite the agreement in trend and minimum switching field angle, the model shows slightly lower absolute normalized switching field values.

In the third case, we introduce a gradual transition of the magnetic composition across the grain boundary in both the soft and hard phases. This may cause an additional incoherency in the soft layer. This model has a core, shell, and 
boundary with radius: $3.5 / 0.25 / 0.25 \mathrm{~nm}$ and material properties as in model 1, except that for the soft and hard layers $K$ and $J_{S}$ of the shell and boundary are $20 \%$ and $10 \%$ of the core, which takes the values of model 1 (see inset Figure 3).

Figure 3(a) shows the comparison of the switching field as function of angle with experiments. The absolute value of the switching field predicted by the model is within $9 \mathrm{mT}$ of the experimental value at an applied field angle of $0^{\circ}$. The switching field values are much closer to the experimental measurements than for model 1 or model 2. Concerning the minimum angle of the angular dependency, we observe the same trend as in model 1 , the minimum is at $45^{\circ}$ except for an exchange value of $0.2 \times 10^{-11} \mathrm{~J} / \mathrm{m}$ where the minimum is at $40^{\circ}$, Figure $3(\mathrm{~b})$. These findings support the existence of the core/boundary region within in the grain as proposed by Lister et al., ${ }^{12}$ and highlight the importance of considering this structure when devising a model to accurately predict the behaviour of real materials.

In conclusion, we have studied the influence of the grain boundary interaction and interface coupling between the soft and hard phase by introducing an exchange transition layer in to our micromagnetic model of exchange coupled composite media. The comparison of the simulations with VSM measurements clearly demonstrates that absolute switching field values can only be explained with a grain boundary model, and that the deviation in angular switching field behaviour from Stoner-Wohlfarth switching can only be explained by the introduction of an interface layer that reduces the coupling between the magnetic phases. The origins of these interface effects are intrinsic to the system itself and are based on diffusion and strain effects due to lattice mismatch and non-atomistic periodicity, as shown by Hrkac et al. ${ }^{1}$ Our results show that as magnetic hard disk drive manufacturers strive to increase areal density by decreasing the diameters of magnetic grains, the magnetic characteristics of the grain boundaries will limit the smallest grain size that can be achieved. This result is significant for the development of Heat Assisted Magnetic Recording (HAMR) systems in which extremely small grain diameters are postulated, and for Bit Patterned Media (BPM) in which discrete patterned islands of sub $10 \mathrm{~nm}$ diameter would be used.

We would like to thank the EPSRC for financial support under Grants No. EP/G032440/1, EP/G032300/1, the WWTF Project MA09-029 and the Royal Society.

${ }^{1}$ G. Hrkac, T. G. Woodcock, C. Freeman, A. Goncharov, J. Dean, T. Schrefl, and O. Gutfleisch, Appl. Phys. Lett. 97, 232511 (2010).

${ }^{2}$ Y. Shinba, T. J. Konno, K. Ishikawa, K. Hiraga, and M. Sagawa, J. Appl. Phys. 97, 053504 (2005).

${ }^{3}$ M. Matsuura, S. Sugimoto, R. Goto, and N. Tezuka, J. Appl. Phys. 105, 07A741 (2009).

${ }^{4}$ R. Dittrich, T. Schrefl, M. Kirschner, D. Suess, G. Hrkac, F. Dorfbauer, O. Ertl, and J. Fidler, IEEE Trans. Magn. 41, 3592 (2005).

${ }^{5}$ R. H. Victora and X. Shen, IEEE Trans. Magn. 41, 2828 (2005).

${ }^{6}$ E. C. Stoner and E. P. Wohlfarth, Philos. Trans. R. Soc. London, Ser. A 240, 599-642 (1948).

${ }^{7}$ E. Kondorsky, J. Phys. (Moscow) 2, 161-181 (1940).

${ }^{8}$ T. Thomson, B. Lengsfield, H. Do, and B. D. Terris, J. Appl. Phys. 103, 07F548 (2008).

${ }^{9}$ T. Schrefl, J. Fidler, R. Dittrich, D. Suess, W. Scholz, V. Tsiantos, and H. Forster, Top. Appl. Phys. 87, 1 (2003).

${ }^{10}$ L. Saharan, C. Morrison, J. J. Miles, T. Thomson, T. Schrefl, and G. Hrkac, J. Appl. Phys. 110, 103906 (2011).

${ }^{11}$ C. Morrison, L. Saharan, G. Hrkac, T. Schrefl, Y. Ikeda, K. Takano, J. J. Miles, and T. Thomson, Appl. Phys. Lett. 99, 132507 (2011).

${ }^{12}$ S. J. Lister, M. P. Wismayer, V. Venkataramana, M. A. De Vries, S. J. Ray, S. L. Lee, T. Thomson, J. Kohlberecher, H. D. Y. Lkeda, K. Takano, and C. Dewhurst, J. Appl. Phys. 106, 063908 (2009).

${ }^{13}$ R. Dittrich, T. Schrefl, D. Suess, W. Scholz, H. Forster, and J. Fidler, J. Magn. Magn. Mater. 250, 12-19 (2002).

${ }^{14}$ T. Schrefl, G. Hrkac, G. Bance, D. Suess, O. Ertl, and J. Fidler, Handbook of Magnetism and Advanced Magnetic Materials (Wiley, New York, 2007), Vol. 2, pp. 765-794.

${ }^{15}$ H. Kronmüller, J. Magn. Magn. Mater. 7, 341 (1978).

${ }^{16}$ D. Suess, L. Breth, J. Lee, M. Fuger, C. Vogler, F. Bruckner, B. Bergmair, T. Huber, J. Fidler, and T. Schrefl, Phys. Rev. B 84, 224421 (2011). 